\title{
Rough Convergence For Difference Sequences
}

\author{
Nihal Demir ${ }^{1}$ and Hafize Gümü̈s ${ }^{2}$ \\ ${ }^{1}$ Necmettin Erbakan University, Institute of Sciences, Konya, Turkey \\ ${ }^{2}$ Necmettin Erbakan University, Department of Mathematical Education, Eregli, Konya, Turkey
}

Received: 12 November 2019, Accepted: 16 February 2020

Published online: 22 June 2020.

\begin{abstract}
As known, difference sequences have their own characteristics. In this paper, we study the concept of rough convergence for difference sequences in a finite dimensional normed space. At the same time, we examine some properties of the set

$$
\operatorname{LIM}_{\Delta x_{i}}^{r}=\left\{x_{*} \in X: \Delta x_{i} \stackrel{r}{\rightarrow} x_{*}\right\}
$$

which is called as $r$-limit set of the difference sequence $\Delta x=\left(\Delta x_{i}\right)$.

Keywords: Convergence, difference sequences, rough convergence, limit points.

\section{Introduction and Background}

It is indisputable that the concept of convergence of a sequence is one of the most important concepts in Summability Theory. Also, determining the place of sequences in that does not satisfy the convergence condition is as important as convergent ones. Although not convergent, the existence of this kind of sequences that show similar characteristics to the concept of convergent sequence under certain conditions, has led to the emergence of different types of convergence. One of these is the concept of rough convergence defined by Phu ([12]) in finite dimensional normed spaces. According to this idea, rough convergence of a sequence can be obtained by extending the range of convergence by a number $r>0$. Here, it should be noted that rough convergence has quite interesting applications in numerical analysis. After Phu's work, Aytar ([3]) studied about rough limit set and the core of a real sequence. Then, Phu ([13]) examined these results in infinite dimensional normed spaces and obtained more general results.
\end{abstract}

Accordingly, the definition of rough convergence in a finite dimensional normed space can be given as follows:

Let $(X,\|\|$.$) be a normed linear space and r$ be a nonnegative real number. Then, the sequence $x=\left(x_{i}\right)$ in $X$ is said to be rough convergent or $r$-convergent to $x_{*}$; if for any $\varepsilon>0$ there exists an $i_{\varepsilon} \in \mathbb{N}$ such that

$$
\left\|x_{i}-x_{*}\right\|<r+\varepsilon
$$

for all $i \geq i_{\varepsilon}$. This expression means that

$$
\limsup \left\|x_{i}-x_{*}\right\|<r
$$

and $r$ is called by roughness degree. In this definition, we say that $x_{*}$ is an $r$-limit point of $\left(x_{i}\right)$ and it is denoted by $x_{i} \stackrel{r}{\rightarrow} x_{*}$. 
Let $\left(x_{i}\right)$ be a rough convergent sequence in a finite dimensional normed space $(X,\|\cdot\|)$ and $r$ be a non-negative real number. For each $r>0$, we obtain a different $x_{*}$ point. So, this point which is called by the $r$-limit point of the sequence is unique. Therefore, a set of these points can be mentioned. This set is called by the set of $r$-limit points and is indicated by $L I M_{x_{i}}^{r}$. As seen, the topological and analytical features of the set are very important. The $r$-limit point set of the sequence $\left(x_{i}\right)$ is defined by

$$
\operatorname{LIM}_{x_{i}}^{r}=\left\{x_{*} \in X: x_{i} \stackrel{r}{\rightarrow} x_{*}\right\}
$$

Phu investigated boundedness and convexity of this set in ([12]). At the same time, he proved that this set is closed.

Following the definition of Phu, the concept of rough convergence was studied by Arslan and Dündar ([1]), Dündar and Çakan ([6]), Dündar([7]) and Kişi and Dündar([10]) for ideal convergence.

Now, lets briefly talk about difference sequences and their main properties. Difference sequences are defined by Kizmaz ([11]) for a real sequence $x=\left(x_{i}\right)$ by $\Delta x=\left(\Delta x_{i}\right)=\left(x_{i}-x_{i+1}\right)$ for all $i \in \mathbb{N}$. He examined the basic properties of $c_{0}(\Delta)$, $c(\Delta)$ and $l_{\infty}(\Delta)$ sequence spaces defined as

$$
\begin{aligned}
& c_{0}(\Delta)=\left\{x=\left(x_{i}\right): \Delta x \in c_{0}\right\} \\
& c(\Delta)=\left\{x=\left(x_{i}\right): \Delta x \in c\right\} \\
& l_{\infty}(\Delta)=\left\{x=\left(x_{i}\right): \Delta x \in l_{\infty}\right\} .
\end{aligned}
$$

In these definitions, $c_{0}, c$ and $l_{\infty}$ are null, convergent and bounded linear sequence spaces, respectively Kizmaz proved that these spaces are Banach spaces by the norm $\|x\|_{\Delta}=\left|x_{1}\right|+\|\Delta x\|_{\infty}$ and he also investigated $\alpha, \beta$ and $\gamma-$ duals of these spaces but we do not interested in the duals in our study. Later on, Aydın and Başar ([2]), Başarır ([4]), Et ([8]), Et and Çolak ([9]) and many others interested in some properties of difference sequences.

\section{Main Results}

After specifying our purpose, let's start by giving the definition of rough convergence for difference sequences in a finite dimensional normed space.

Definition 1. Let $(X,\|\|$.$) be a normed space, r$ be a non-negative real number and $\left(\Delta x_{i}\right)$ be a difference sequence in $X$. For every $\varepsilon>0$ and $i \geq i_{\varepsilon}$; if there is an $i_{\varepsilon}$ such that

$$
\left\|\Delta x_{i}-x_{*}\right\|<r+\varepsilon
$$

or equivalently

$$
\limsup _{i \rightarrow \infty}\left\|\Delta x_{i}-x_{*}\right\| \leq r
$$

then, the sequence $\left(\Delta x_{i}\right)$ is rough convergent to $x_{*}$, where $\Delta x=\left(\Delta x_{i}\right)=\left(x_{i}-x_{i+1}\right)$. We denote $r$-limit set of $\left(\Delta x_{i}\right)$ by

$$
\operatorname{LIM}_{\Delta x_{i}}^{r}=\left\{x_{*} \in X: \Delta x_{i} \stackrel{r}{\rightarrow} x_{*}\right\}
$$

If we obtain a new type of convergence, it would be interesting to compare this type of convergence with the known types of convergence. We can explain this comparison with some examples. The first example is an example of a difference sequences that are not convergent but $r$-convergent. 
Example 1.Take the sequence $x_{i}=\left\{\begin{array}{l}2, \text { if } i \text { is odd } \\ 1, \text { if } i \text { is even }\end{array}\right.$. Then $\left(\Delta x_{i}\right)=(-1)^{i}$ and we can easily say that $\left(\Delta x_{i}\right)$ is not convergent but $r$-convergent. Because, from the definition 2.1, if $\left(\Delta x_{2 i}\right)=(1,1,1,1, \ldots)$, then for every $\varepsilon>0$

$$
\begin{aligned}
-r-\varepsilon<1-x_{*}<r+\varepsilon & \Longrightarrow 1-r-\varepsilon<x_{*}<1+r+\varepsilon \\
& \Longrightarrow x_{*} \in[1-r, 1+r]
\end{aligned}
$$

and if $\left(\Delta x_{2 i-1}\right)=(-1,-1,-1,-1, \ldots)$, then for every $\varepsilon>0$

$$
\begin{aligned}
-r-\varepsilon<-1-x_{*}<r+\varepsilon & \Longrightarrow-1-r-\varepsilon<x_{*}<-1+r+\varepsilon \\
& \Longrightarrow x_{*} \in[-1-r,-1+r]
\end{aligned}
$$

and so

$$
\operatorname{LIM}_{\Delta x_{i}}^{r}=\left\{\begin{array}{ll}
\emptyset, & \text { if } r<1 \\
{[1-r,-1+r]} & \text { if } r \geq 1
\end{array} .\right.
$$

This result gives us the $r$-convergence of $\left(\Delta x_{i}\right)$.

The sequence given in the second example is both convergent and $r$-convergent.

Conclusion Let $\left(\Delta x_{i}\right)=\left(1+\frac{1}{i}\right)$. Then $\left(\Delta x_{i}\right)$ is both convergent and $r$-convergent. Because

$$
\begin{aligned}
-r-\varepsilon+\Delta x_{i}<x_{*}<r+\varepsilon+\Delta x_{i} & \Longrightarrow-r-\varepsilon+1+\frac{1}{i}<x_{*}<r+\varepsilon+1+\frac{1}{i} \\
& \Longrightarrow \text { for every } \varepsilon>0, \frac{1}{i} \rightarrow 0, x_{*} \in[1-r, 1+r]
\end{aligned}
$$

and so

$$
\operatorname{LIM}_{\Delta x_{i}}^{r}=[1-r, 1+r] .
$$

If $L I M_{\Delta x_{i}}^{r} \neq \emptyset$, then $\operatorname{LIM}_{\Delta x_{i}}^{r}=\left[\lim \sup \Delta x_{i}-r, \liminf \Delta x_{i}+r\right]$.

As is known, in the classical sense, a convergent sequence has a single limit and each subsequence of the sequence converges to the same point. The following theorems will explain how these states will find a response for difference sequences and rough convergence.

Theorem 1. For any difference sequence $\Delta x=\left(\Delta x_{i}\right)$, diameter of $\operatorname{LIM}_{\Delta x_{i}}^{r}$ is not greater than $2 r$. Generally, there is no smaller bound.

Proof. If we show that

$$
\operatorname{diam}\left(\operatorname{LIM}_{\Delta x_{i}}^{r}\right)=\sup \left\{\|y-z\|: y, z \in \operatorname{LIM}_{\Delta x_{i}}^{r}\right\} \leq 2 r,
$$

then we will have the proof.

Suppose that $\operatorname{diam}\left(\operatorname{LIM}_{\Delta x_{i}}^{r}\right)>2 r$. Then there exists $y, z \in L I M_{\Delta x_{i}}^{r}$ such that

$$
d:=\|y-z\|>2 r
$$

and for an arbitrary $\varepsilon \in\left(0, \frac{d}{2-r}\right)$ there exist an $i_{0} \in \mathbb{N}$ such that

$$
\left\|\Delta x_{i}-y\right\|<r+\varepsilon
$$


and

$$
\left\|\Delta x_{i}-z\right\|<r+\varepsilon
$$

for $i \geq i_{0}$. In this case, we obtain

$$
\|y-z\| \leq\left\|\Delta x_{i}-y\right\|+\left\|\Delta x_{i}-z\right\|<2(r+\varepsilon)<2 r+2\left(\frac{d}{2-r}\right)=d
$$

but this result contradicts with $d:=\|y-z\|$. So, $\operatorname{diam}\left(\operatorname{LIM}_{\Delta x_{i}}^{r}\right) \leq 2 r$ is true.

Now, let's show that there is generally no smaller bound. For this, we show that $\operatorname{LIM}_{\Delta x_{i}}^{r}=\bar{B}_{r}\left(x_{*}\right)$. We know that $\operatorname{diam} \bar{B}_{r}\left(x_{*}\right)=2 r$ for

$$
\bar{B}_{r}\left(x_{*}\right):=\left\{y \in X:\left\|y-x_{*}\right\| \leq r\right\} .
$$

Choose a convergent difference sequence $\left(\Delta x_{i}\right)$ with $\lim \Delta x_{i}=x_{*}$. For each $\varepsilon>0$ and for all $i \geq i_{0}$, there is an $\exists i_{0} \in \mathbb{N}$ such that $\left\|\Delta x_{i}-x_{*}\right\|<\varepsilon$.

$$
\left\|\Delta x_{i}-y\right\| \leq\left\|\Delta x_{i}-x_{*}\right\|+\left\|x_{*}-y\right\| \leq\left\|\Delta x_{i}-x_{*}\right\|+r,\left(\text { for } y \in \bar{B}_{r}\left(x_{*}\right)\right)
$$

and from the definition of rough limit point set we have $\operatorname{LIM}_{\Delta x_{i}}^{r}=\bar{B}_{r}\left(x_{*}\right)$.

Theorem 2. A difference sequence $\left(\Delta x_{i}\right)$ is bounded if and only if there exists an $r \geq 0$ such that $\operatorname{LIM}_{\Delta x_{i}}^{r} \neq \emptyset$.

Proof. Assume that $L I M_{\Delta x_{i}}^{r} \neq \emptyset$ and $s:=\sup \left\{\left\|\Delta x_{i}\right\|: i \in \mathbb{N}\right\}<\infty$ for some $r \geq 0$. Then LIM $_{\Delta x_{i}}^{s}$ contain the origin of $X$. On the other hand; if $L I M_{\Delta x_{i}}^{r} \neq \emptyset$ for some $r \geq 0$, then all $\Delta x_{i}$ except finite elements are contained in some ball with any radius greater then $r$. So, the sequence $\left(\Delta x_{i}\right)$ is bounded.

Now, suppose that $\left(\Delta x_{i}\right)$ is bounded. In this case it is clear that it has a convergent subsequence $\left(\Delta x_{i_{j}}\right)$. Let $x_{*}$ be the limit point of this subsequence. Then, $\operatorname{LIM}_{\Delta x_{i_{j}}}^{r}=\bar{B}_{r}\left(x_{*}\right)$ and for $r>0$,

$$
\operatorname{LIM}^{\left(\Delta x_{i_{j}}\right), r} \Delta x_{i_{j}}=\left\{\Delta x_{i_{j}}:\left\|x_{*}-\Delta x_{i_{j}}\right\| \leq r\right\} \neq 0 .
$$

As is known, each subsequence of a convergent sequence converges to the same limit point. Similarly, we have the following theorem for rough convergent difference sequences.

Theorem 3. If $\left(\Delta x_{i_{j}}\right)$ is a subsequence of the difference sequence $\left(\Delta x_{i}\right)$ then, $\operatorname{LIM}_{\Delta x_{i}}^{r} \subseteq \operatorname{LIM}_{\Delta x_{i_{j}}}^{r}$.

Proof. Suppose that $\left(\Delta x_{i_{j}}\right)$ is a subsequence of the difference sequence $\left(\Delta x_{i}\right)$ and $x_{*} \in \operatorname{LIM}_{\Delta x_{i}}^{r}$. In this instance,

$$
\left\|\Delta x_{i}-x_{*}\right\|<r+\varepsilon
$$

and

$$
\left\|\Delta x_{i_{j}}-x_{*}\right\|<r+\varepsilon
$$

for $i \in \mathbb{N}$ which means $x_{*} \in \operatorname{LIM}_{\Delta x_{i_{j}}}^{r}$. Then $\operatorname{LIM}_{\Delta x_{i}}^{r} \subseteq \operatorname{LIM}_{\Delta x_{i_{j}}}^{r}$.

It is also important to know the geometric and topological properties of the set of limit points. These properties will be explained in the theorems given below. 
Theorem 4. For an arbitrary difference sequence $\left(\Delta x_{i}\right)$ and for all $r \geq 0$ the set LIM ${ }_{\Delta x_{i}}^{r}$ is closed.

Proof. We will use a theorem which is well known in functional analysis for this proof "Let $y=\left(y_{j}\right) \in c(\Delta)$ is a $\Delta$-convergent sequence and $\Delta y_{j} \rightarrow y_{*}$. When $y \in \operatorname{LIM}_{\Delta x_{i}}^{r}$ is also $y_{*} \in L_{I M_{\Delta x_{i}}^{r}}$, then the set $\operatorname{LI}_{\Delta x_{i}}^{r}$ is closed."

Now, assume that the sequence $y=\left(y_{j}\right) \in c(\Delta), \Delta y_{j} \rightarrow y_{*}$ and $y \in L I M_{\Delta x_{i}}^{r}$. For every $\varepsilon>0$ and for $i \geq i_{\varepsilon / 2}$ there are a $j_{\varepsilon / 2}$ and an $i_{\varepsilon / 2}$ such that

$$
\left\|\Delta y_{j_{\varepsilon / 2}}-y_{*}\right\|<\frac{\varepsilon}{2}
$$

and

$$
\left\|\Delta x_{i}-\Delta y_{j_{\varepsilon / 2}}\right\|<r+\frac{\varepsilon}{2}
$$

For every $i \geq i_{\varepsilon / 2}$,

$$
\left\|\Delta x_{i}-y_{*}\right\| \leq\left\|\Delta x_{i}-\Delta y_{j_{\varepsilon / 2}}\right\|+\left\|\Delta y_{j_{\varepsilon / 2}}-y_{*}\right\|<r+\varepsilon
$$

and so $y_{*} \in L I M_{\Delta x_{i}}^{r}$.

Theorem 5. (a) If $y_{0} \in \operatorname{LIM}_{\Delta x_{i}}^{r_{0}}$ and $y_{1} \in \operatorname{LIM}_{\Delta x_{i}}^{r_{1}}$ then $y_{\lambda}:=(1-\lambda) y_{0}+\lambda y_{1} \in \operatorname{LIM}_{\Delta x_{i}}^{(1-\lambda) r_{0}+\lambda r_{1}}$ for $\lambda \in[0,1]$.

(b) The set LIM ${ }_{\Delta x_{i}}$ is convex.

Proof.

(a) Assume that $y_{0} \in \operatorname{LIM}_{\Delta x_{i}}^{r_{0}}$ and $y_{1} \in L I M_{\Delta x_{i}}^{r_{1}}$. In this case, for every $\varepsilon>0$ there exists an $i_{\varepsilon}$ such that $i \geq i_{\varepsilon}$ implies $\left\|\Delta x_{i}-y_{0}\right\|<r_{0}+\varepsilon$ and $\left\|\Delta x_{i}-y_{1}\right\|<r_{1}+\varepsilon$ which yields also

$$
\begin{aligned}
\left\|\Delta x_{i}-y_{\lambda}\right\| & \leq(1-\lambda)\left\|\Delta x_{i}-y_{0}\right\|+\lambda\left\|\Delta x_{i}-y_{1}\right\| \\
& <(1-\lambda)\left(r_{0}+\varepsilon\right)+\lambda\left(r_{1}+\varepsilon\right) \\
& =(1-\lambda) r_{0}+\lambda r_{1}+\varepsilon
\end{aligned}
$$

Then, we have $y_{\lambda} \in L I M_{\Delta x_{i}}^{(1-\lambda) r_{0}+\lambda r_{1}}$.

(b) If we choose $r=r_{0}=r_{1}$ in $(a)$ it is easily seen that $L I M_{\Delta x_{i}}^{r}$ is convex.

The following theorem formulates an additive property of rough convergence with difference sequences.

Theorem 6. Let $r_{1} \geq 0$ and $r_{2} \geq 0 .\left(\Delta x_{i}\right)$ is $\left(r_{1}+r_{2}\right)$-convergent to $x_{*}$ if and only if there exists a difference sequence $\left(\Delta y_{i}\right)$ such that

$$
\Delta y_{i} \stackrel{r_{1}}{\rightarrow} x_{*} \text { and }\left\|\Delta x_{i}-\Delta y_{i}\right\| \leq r_{2}(i \in \mathbb{N})
$$

Proof. Suppose that $\Delta y_{i} \stackrel{r_{1}}{\rightarrow} x_{*}$ and $\left\|\Delta x_{i}-\Delta y_{i}\right\| \leq r_{2}$. Then, for every $\varepsilon>0$ and $i \geq i_{\varepsilon}$ there exists an $i_{\varepsilon}$ such that

$$
\left\|\Delta y_{i}-x_{*}\right\| \leq r_{1}+\varepsilon
$$

From $\left\|\Delta x_{i}-\Delta y_{i}\right\| \leq r_{2}$, we have

$$
\left\|\Delta x_{i}-x_{*}\right\| \leq\left\|\Delta x_{i}-\Delta y_{i}\right\|+\left\|\Delta y_{i}-x_{*}\right\|<r_{1}+r_{2}+\varepsilon
$$


if $i \geq i_{\varepsilon}$. So, $\left(\Delta x_{i}\right)$ is $\left(r_{1}+r_{2}\right)-$ convergent to $x_{*}$.

Now, assume that $\Delta x_{i} \stackrel{r_{1}+r_{2}}{\rightarrow} x_{*}$ and let's try to show that $\left\|\Delta y_{i}-x_{*}\right\| \leq r_{1}$ and $\left\|\Delta x_{i}-\Delta y_{i}\right\| \leq r_{2}$ for $i \geq i_{\varepsilon}$. With

$$
\Delta y_{i}:=\left\{\begin{array}{ll}
x_{*} & \text { if }\left\|\Delta x_{i}-x_{*}\right\| \leq r_{2} \\
\Delta x_{i}+r_{2} \frac{x_{*}-\Delta x_{i}}{\left\|x_{*}-\Delta x_{i}\right\|} & , \text { if }\left\|\Delta x_{i}-x_{*}\right\|>r_{2}
\end{array},\right.
$$

we have

$$
\left\|\Delta y_{i}-x_{*}\right\| \leq \begin{cases}0 & , \text { if }\left\|\Delta x_{i}-x_{*}\right\| \leq r_{2} \\ \left\|x_{i}-x_{*}\right\| & , \text { if }\left\|\Delta x_{i}-x_{*}\right\|>r_{2}\end{cases}
$$

and

$$
\left\|\Delta x_{i}-\Delta y_{i}\right\| \leq r_{2}
$$

for $i \in \mathbb{N}$. At the same time, we know that $\Delta x_{i} \stackrel{r_{1}+r_{2}}{\rightarrow} x_{*}$ implies

$$
\limsup \left\|\Delta x_{i}-x_{*}\right\| \leq r_{1}+r_{2}
$$

So,

$$
\limsup \left\|\Delta y_{i}-x_{*}\right\| \leq r_{1}
$$

and we have the proof.

Theorem 7. A sequence $\left(\Delta x_{i}\right) \in \mathbb{R}^{n}$ convergent to $x_{*}$ if and only if LIM $\operatorname{Lx}_{i}=\bar{B}_{r}\left(x_{*}\right)$ where $\bar{B}_{r}\left(x_{*}\right):=\left\{y \in X:\left\|y-x_{*}\right\| \leq r\right\}$.

Proof. If $\Delta x_{i} \rightarrow x_{*}$, then we have $\operatorname{LIM}_{\Delta x_{i}}^{r}=\bar{B}_{r}\left(x_{*}\right)$.

Now, assume that $L I M_{\Delta x_{i}}^{r}=\bar{B}_{r}\left(x_{*}\right)$ and $\left(\Delta x_{i}\right)$ has a cluster point $y_{*}$ different from $x_{*}$. Then the point

$$
\bar{x}_{*}:=x_{*}+\frac{r}{\left\|x_{*}-y_{*}\right\|}\left(x_{*}-y_{*}\right)
$$

satisfies

$$
\left\|\bar{x}_{*}-y_{*}\right\|=r+\left\|x_{*}-y_{*}\right\|>r .
$$

From the fact that $y_{*}$ is a cluster point, the last inequality implies that $\bar{x}_{*} \notin L I M_{\Delta x_{i}}^{r}$ and this contradicts with $\left\|\bar{x}_{*}-y_{*}\right\|=r$ and $L I M_{\Delta x_{i}}^{r}=\bar{B}_{r}\left(x_{*}\right)$. So, our assumption is wrong and $x_{*}$ is the only cluster point of the sequence. Then, $\Delta x_{i} \rightarrow x_{*}$.

\section{Conclusions}

As we will see from many studies, difference sequences have their own characteristics. For example, it is easy to see that $c \subseteq c(\Delta)$. Therefore, in this article, it was interesting to see the results obtained when the concept of rough convergence is studied for difference sequences.

\section{Acknowledgements}

The authors are grateful to the referees and the editor for their corrections and suggestions, which have greatly improved the readability of the paper. 


\section{Competing interests}

The authors declare that they have no competing interests.

\section{Authors' contributions}

All authors have contributed to all parts of the article. All authors read and approved the final manuscript.

\section{References}

[1] Arslan, M. and Dündar, E., On rough convergence in 2-normed spaces and some properties, Filomat, 33 (16), 157(3), 5077-5086 (2019).

[2] Aydın, C. and Başar, F., Some new difference sequence spaces, Appl. Math.Comput. 157(3), 677-693 (2004).

[3] Aytar, S., The Rough Limit Set and the Core of a Real Sequence, Numer. Func. Anal. Optimiz. 29, No 3, 283-290 (2008).

[4] Basarir, M., On the $\Delta$ - statistical convergence of sequences, Firat Uni., Jour. of Science and Engineering 7(2), 1-6 (1995).

[5] Demir, N., Rough convergence and rough statistical convergence of difference sequences, Master Thesis in Necmettin Erbakan University, Institue of Natural and Applied Sciences, June 2019.

[6] Dündar, E. and Çakan, C., Rough $\mathscr{I}$-convergence, Demonstratio Mathematica 2(1) (2014) 45-51.

[7] Dündar, E., On Rough $\mathscr{I}_{2}$ - convergence, Numer. Funct. Anal. and Optimiz. 37(4), 480-491 (2016).

[8] Et, M., On some difference sequence spaces, Doğa-Tr. J. of Mathematics 17, 18-24 (1993).

[9] Et, M. and Çolak, R., On some generalized difference sequence spaces, Soochow Journal Of Mathematics, 21(4), $377-386$ (1995).

[10] Kişi, Ö. and Dündar, E., Rough $\mathscr{I}_{2}$-lacunary statistical convergence of double sequences , Journal of Inequalities and Applications, 2018:230, 16 pages, https://doi.org/10.1186/s13660-018-1831-7 (2018).

[11] Kizmaz, H., On certain sequence spaces, Canad. Math. Bull. 24(2), 169-176 (1981).

[12] Phu, H. X., Rough convergence in normed lineer spaces, Numer. Funct. Anal.Optmiz., Vol. 22, 199-222 (2001).

[13] Phu, H. X., Rough Convergence infinite dimensional normed spaces, Journal of Numerical Functional Analysis and Optimization, Vol.24, 285-301 (2003). 